\title{
Expression and Purification of Soluble form of Human Parathyroid Hormone (rhPTH1-34) by Trx Tag in E. coli
}

\author{
Sanaz Yari, Farida Behzadian*, Hamideh Rouhani Nejad, Mohammadreza Masoumian, Mahdi Karimi \\ Department of Biosciences and Biotechnology, Malek-Ashtar University of Technology, Tehran, Iran.
}

Received: 11 Jul 2017

Revised : 20 Aug 2017

Accepted: 10 Sep 2017

Corresponding Author:

Farida Behzadian

Department of Biosciences and

Biotechnology, Malek-Ashtar

University of Technology, Tehran, Iran.

Phone: +98-2122974603

E-mail: fbehzadian@yahoo.com

\begin{abstract}
Background: Parathyroid Hormone (PTH) is secreted by parathyroid glands and controls the level of calcium in bones and kidney. PTH is a small polypeptide with 84 amino acids, but the first 34 amino acids of which are enough for hormone biological activity and can be used in the treatment of Osteoporosis. The expression efficiency of recombinant human parathyroid hormone rhPTH (1-34) or Teriparatide using a cleavable fusion protein strategy was compared in two strains of E. coli.

Materials and Methods: A cassette was designed and fully synthesized for prokaryotic expression of rhPTH using pET system. From 5' to 3', the cassette consisted of: Trx tag to increase the solubility of protein, His tag for purification and detection of protein, enterokinase site to cleave all fusion moieties, and an optimized gene code for Teriparatide corresponding to the amino acid sequence of $\mathrm{hPTH}$. This cassette was cloned into pET32a vector. The vector was simultaneously transformed and expressed in two different E. coli strains. The ability of strains for expression of this recombinant pharmaceutical was compared. Early expression was confirmed by SDS-PAGE and Western Blotting. The soluble fusion protein was harvested and purified by immobilized affinity chromatography. Then the fusion moiety was released from Teriparatide by enterokinase digestion.

Results: The fusion form of rhPTH was efficiently expressed in both E. coli strains. However, the percentage of the target protein to the total protein content in Rosetta-gami was more than its amount in BL21 (60\% vs 25\%).The fusion protein was highly purified with Ni-NTA column. Up to $18.5 \mathrm{mg} / \mathrm{ml}$ of pure fusion protein has been obtained from 1-liter Rosetta-gami strain of E. coli. The pure Teriparatide was released by enterokinase digestion.

Conclusion: The pure rhPTH (1-34) produced here, could be the subject for biological activity and quality control assessments, and following formulation processing, it could be applied as a peptide drug in the treatment of Osteoporosis.
\end{abstract}

Keywords: Enterokinase; Fusion protein; Parathyroid hormone; BL21; Rosettagami

Please cite this article as: Yari S, Behzadian F, Rouhani Nejad H, Masoumian M, Karimi M. Expression and Purification of Soluble form of Human Parathyroid Hormone (rhPTH1-34) by Trx Tag in E. coli. Res Mol Med. 2017; 5 (3): 26-31

\section{Introduction}

Teriparatide or rhPTH (1-34) is the recombinant bioactive portion of human parathyroid hormone secreted by parathyroid glands. Parathyroid hormone (PTH) has 115 amino acids in its original form; the first $25 \mathrm{~N}$-terminal amino acids are separated as a signal, the next 6 amino acids are separated during secretion from Golgi, and finally, the mature form is secreted from parathyroid glands with 84 amino acids. This hormone affects the metabolism of calcium and phosphorus and acts directly on the kidneys and bone tissues(1). Studies show that all these 84 amino acids are not necessary for the 
functioning of hormone and the main biological activity is related to N-terminal 1-34 amino acids (2). PTH binds to specific receptors in osteoblasts and renal tubular cells and results in upregulation of RANKL expression, a protein essential for osteoclast development and survival $(3,4)$. PTH signaling occurs via a PTH receptor 1 , cAMP, and protein kinase $\mathrm{A}$, CREB cascade. Moreover, this hormone indirectly increases serum level of calcium through its effects on metabolism of 1,25 di-hydroxi vitamin D (5).

Teriparatide has been FDA approved since 2002 and has been used as a drug to treat patients with Osteoporosis (4). Several studies used different hosts for expression of Teriparatide, such as E. coli, yeasts, and even mammalian cells (6). Since the molecular weight of this recombinant hormone is low (4 kDa), the risk of protein digestion by intracellular proteases is very high at the time of expression (7). To solve this problem, the polypeptide is expressed in fusion with another protein as part of a partner; this increase in size inhibits the protease activity of the protein expression. Different partners are used to express Teriparatide, such as Saccharomyces cerevisiae V8, Fc in human IgG, etc. (8). In this study, Trx tag was used as partner, which in addition to the aforementioned features has unique characteristics that could play an important role to optimize its expression. Trx tag, weighing about $12 \mathrm{kDa}$, helps folding of the fusion protein with the creation of disulfide bonds, and prevents the formation of inclusion bodies, which is one of the problems of cytoplasmic expression in prokaryotic hosts and changes proteins to cytoplasmic solution form $(9,10)$. This unique feature enhances the quality of expression efficiency in downstream processes, such as purification, and omission of the de-urea and folding, along with reducing the process of purification steps, increases the final efficiency and prevents the loss of protein. The most generally used host organism for biopharmaceutical production is E. coli. BL21 and Rosetta-gami strains of E. coli are the most popular prokaryotic hosts for expression of recombinant proteins (11).

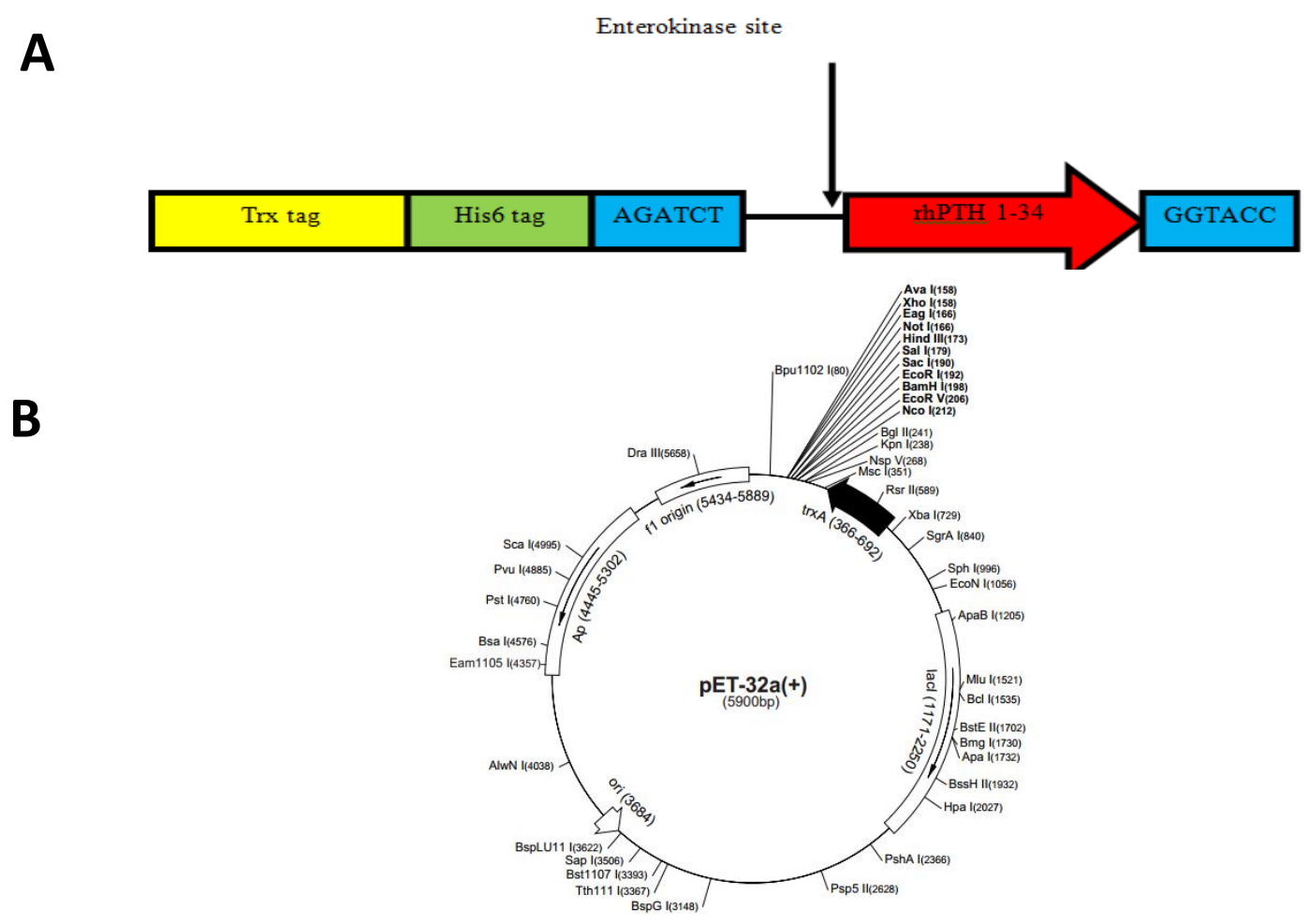

Figure 1. A) Expression cassette of rhPTH 1-34 which codes for a fusion protein with molecular weight of 22kDa B) The restriction map of pET-32a.The position of restriction sites through which cloning is carried out.

\section{Materials and methods}

Construction of the expression vector for the prokaryotic expression of recombinant hormone, expression cassette of pET32a was designed and fully synthesized (Pouya Gene AZMA Co, IRAN). As shown in Figure 1, this cassette from 5' to 3' includes
Trx tag (between BglII and KpnI) to enhance the solubility of recombinant protein, His tag for purification and a specific restriction site for enterokinase that separates connected tags from the original protein structure. The abov-mentioned cassette was cloned into pET32 vector and expressed 
in two strains of $E$. coli.

Expression of rhPTH 1-34 in different E. coli hosts The rhPTH-pET plasmid was transformed into $E$. coli Rosetta-gami and BL21 via transformation. Single colonies from transformed cells were used for inoculation of $5 \mathrm{ml}$ pre-culture medium containing ampicillin $(100 \mathrm{mg} / \mathrm{ml})$. The cultures were incubated for $24 \mathrm{hr}$ at $37{ }^{\circ} \mathrm{C}$ with the shake of $180 \mathrm{rpm} .250 \mu \mathrm{l}$ of the pre-cultures were used for inoculation of $5 \mathrm{ml}$ of LB medium (ampicillin $100 \mathrm{mg} / \mathrm{ml}$ ) at $37{ }^{\circ} \mathrm{C}$ until reaching the OD 600 of 0.7 . For protein expression, Isopropyl $\beta$-D-1-thiogalactopyranoside (IPTG) was added to the final concentration of $1 \mathrm{mM}$. Expression was continued at $37{ }^{\circ} \mathrm{C}$ for $4 \mathrm{hr}$ and cells were harvested within desired intervals. Quantification of protein expression released in medium was carried out by image analysis of the SDS-PAGE in 14.5 percent scans using CLIQS (12).

\section{Western blotting}

Using specific antibody-antigen binding, recombinant protein can be detected. The samples were transferred from the gel onto nitrocellulose paper. After transfer, the paper was incubated for an hour in BSA 3\% solution for blocking at room temperature with shake. Afterward, the paper was washed three times with TBS-T solution and was reacted with Anti-His6 (Roche Cat\# 04905318001) diluted 1:6000 in TBS-T for $1 \mathrm{hr}$ at room temperature. After repeated washing of paper, it was incubated with the appropriate secondary antibody, anti-mouse IgG Peroxidase conjugate (Sigma Cat\# A8924) diluted 1:10000 in TBS-T at the same condition. Then, rinsing was repeated and, finally, by adding a solution of 3, 3'- diaminobenzidine (DAB) (Sigma Cat\# D5637) in the darkness after about 3 minutes, the bands related to the recombinant protein were observed in the expected size.

\section{Purification of recombinant PTH}

The frozen biomass of $E$. coli was resuspended in washing buffer $(50 \mathrm{mM} \mathrm{Na} 2 \mathrm{HPO} 4 / \mathrm{NaH} 2 \mathrm{Po} 4-\mathrm{pH}=$ 7.4). After the centrifuge (9000rpm for $5 \mathrm{~min}$ ), the resulting cells were resuspended in lysis buffer (20 $\mathrm{mM}$ Tris, $0.5 \mathrm{M} \mathrm{NaCl}, 10 \mathrm{mM}$ Imidazole, PMSF $1 \mathrm{mM}-\mathrm{pH}=8)$. Then, sonication was performed in $100 \mathrm{~W}$ at $4{ }^{\circ} \mathrm{C}(12$ cycles, 10 seconds off and 15 seconds on) and centrifuged at 11,000 rpm. The supernatant containing the fusion protein was collected and applied to Ni-NTA resin (Chelating Sepharose Fast Flow). The column was equilibrated with buffer A $(20 \mathrm{mM}$ Tris, $0.5 \mathrm{M} \mathrm{NaCl}, 20 \mathrm{mM}$ Imidazole-pH=8) followed by washing with buffer $\mathrm{A}$. The fusion protein was eluted with Linear gradient of Imidazole concentration by Gradient maker with buffer B $(20 \mathrm{mM}$ Tris, $0.5 \mathrm{M}$ Nacl, $500 \mathrm{mM}$ Imidazole $\mathrm{pH}=8$ ). Fractions containing His6Thioredoxin-hPTH (1-34) fusion protein were pooled and were evaluated by SDS-PAGE gel.

Proteolytic cleavage of fusion protein with enterokinase

In order to cleave hPTH from its tag, the desalted fusion protein by dialysis process was digested with enterokinase enzyme (EK MAX, Invitrogen Co, USA) for $16 \mathrm{hr}$ at room temperature. After 16 hours, the results of the digestion were evaluated by SDSPAGE gel.

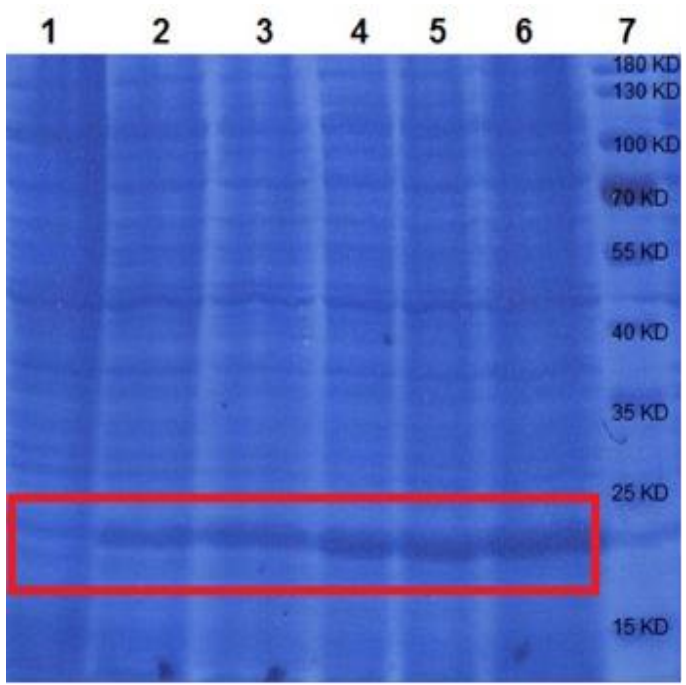

Figure 2. A) Expression of rhPTH 1-34 in Rosetta- gami: Lane 1; before induction. Lanes 2, 3, 4, 5 and 6 were shown $30 \mathrm{~min}, 1 \mathrm{hr}, 2 \mathrm{hr}, 3 \mathrm{hr}$ and 4hr after induction, respectively; Lane 7, protein marker. B) Expression of rhPTH1-34 in BL21: lane 1 before induction; Lanes 2, 3, 4, 5 and 6 were shown $30 \mathrm{~min}, 1 \mathrm{hr}, 2 \mathrm{hr}, 3 \mathrm{hr}$ and $4 \mathrm{hr}$ after induction, respectively; Lane 7, protein marker. 


\section{Results}

Construct and expression of recombinant hPTH with Trx tag

The gene sequence encoded for PTH (1-34) was inserted in the $\mathrm{C}$ terminal end of thioredoxin gene sequence. Between them, enterokinase specific recognition site of (Asp-Asp-Asp-Asp-Lys) was inserted as a specific recognition site. The construct was inserted in pET32a $(+)$. The expression vector was transformed into two $E$. coli strains, including BL21 and Rosetta-gami.

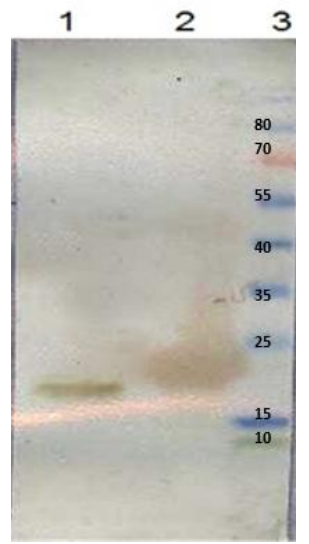

Figure 3. Western blotting for rhPTH protein+Trx tag. Lane 1, a positive control (interferon alpha fused with His-tag). Lane 2, rhPTH protein+ Trx tag expression in the Rosetta-gami. Lane 3, Mw marker.
The over -expression was obtained $4 \mathrm{hr}$ after IPTG $(1 \mathrm{Mm})$ induction in the Rosetta-gami. The results shown in Figure 2 are related to the expression in the BL21 and Rosetta-gami. The Results of CLIQS analysis showed that the yields of expression $4 \mathrm{hr}$ after induction are $45 \%$ and $23 \%$ of the total protein in the Rosetta-gami and BL21 respectively.

Confirm the expression of rhPTH protein + Trx tag by Western blotting

Western blotting was performed before purification for confirmation of rhPTH1-34+Trx tag recombinant protein $(22 \mathrm{kDa})$. Figure 3 is related to expression of target protein in Rosetta-gami.

Purification of rhPTH + Trx tag

The rhPTH + Trx tag proteins were applied to NiNTA chromatography. The purified protein was obtained by $70 \%$ concentration in the supernatant (Figure 4A). Pattern of the eluted PTH protein from the Imidazol gradient (Figure 4B).

\section{Cleavage of the rhPTH from Trx tag}

The Trx tag (18 kDa) was detached from rhPTH through digestion with enterokinase (Figure 5). Two bands were determined including Trx tag (18 kDa) and $\mathrm{rhPTH}(4 \mathrm{kDa})$ in the SDS-PAGE.

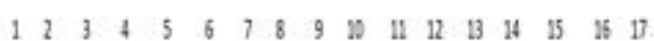

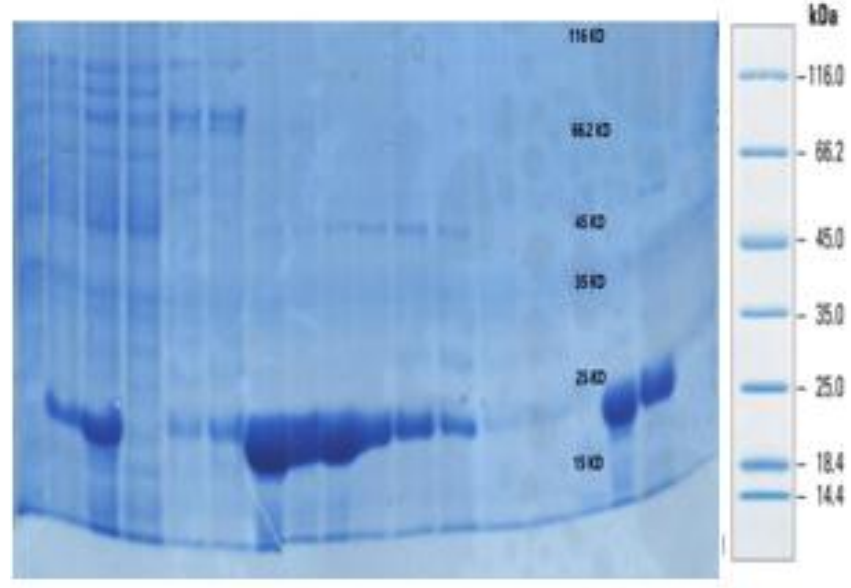

A

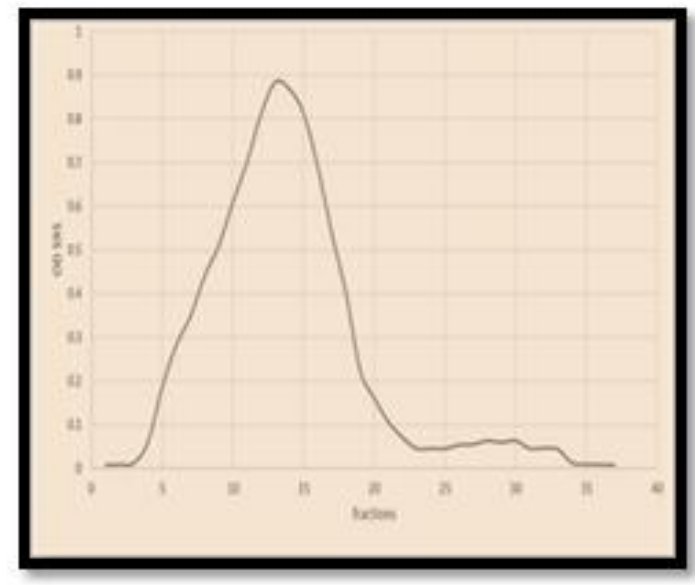

$B$

Figure 4. A) SDS-PAGE for fraction of outlet Ni-NTA chromatography. Lane 1, before induction. Lane 2, second supernatant. Lane 3, first supernatant. Lane 4, out let. Lane 5, fraction 7. Lane 6, fraction 8. Lane 7, fraction 14. Lane 8, fraction 15. Lane 9, fraction 16. Lane 10, fraction 18. Lane 11, fraction 19. Lane 12, fraction 20. Lane 13, fraction 26. Lane 14, fraction 27. Lane 15, MW. Lane 16, pooled fraction (11-16). Lane 17, pooled fraction (17-22). B) Profile of the eluted PTH protein from the Imidazole gradient. The best concentration can be seen in 14-22.

Cleavage of the rhPTH from Trx tag

The Trx tag $(18 \mathrm{kDa})$ was detached from rhPTH through digestion with enterokinase (Figure 5). Two bands were determined including Trx tag $(18 \mathrm{kDa})$ and $\operatorname{rhPTH}(4 \mathrm{kDa})$ in the SDS-PAGE. 
1

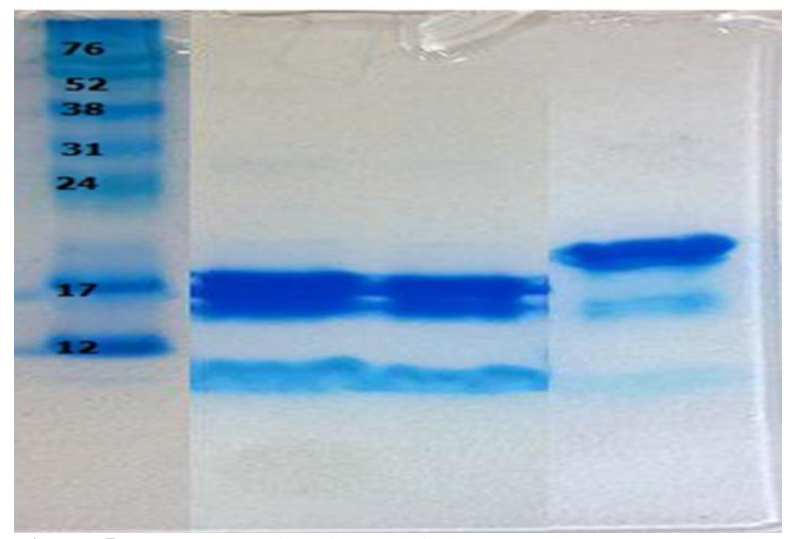

Figure 5. SDS-PAGE for digested fusion protein. Lane 1, Protein Ladder; Lanes 2 and 3, two same samples from digested protein rhPTH $(4 \mathrm{kD})$ and Trx tag $(18 \mathrm{kD})$; and lane 4 is undigested PTH fusion protein $(22 \mathrm{kD})$.

\section{Discussion}

Osteoporosis is a metabolic disorder due to bone tissue loses its strength and becomes highly sensitive to be broken. According to the reports, one in every two women and one in every four men over age 50 years old persons are suffering from this disease. The presence of this disease in Iran considerably increased from $15 \%$ of the population in 2001 to $34 \%$ in 2011. Based on the reports, $20 \%$ of Iranian women over 50 years old are affected by remarkable degrees of bone density reduction. Due to the significance of this disease and its high cost of therapy, using new treatment methods is clearly important.

In 2002, a human recombinant drug, Teriparatide (commercially named FORTEO), was produced by Lilly pharmaceutical company and released to the customer market for Osteoporosis therapy (13). This hormone is also the only effective anabolic drug against Osteoporosis that also is currently consumed in different countries such as Iran. The need for this drug in Iran will be increased in the near future because of the growing elderly population and life expectancy.

In 1991, Forsberg et al surveyed on expression of this hormone in BL21 E. coli that was fused to the carboxylic end of $15 \mathrm{KD}$ human IgG binding protein. They reported that, the final yield of this hormone was $5 \mathrm{mg}$ in each 1 liter of culture media (14). Oshika et al in 1994 studied the direct expression of this polypeptide in E. coli which was $27 \mathrm{mg}$ per liter based on their report (15). In the recent study that was done in 2017 by Bakhtiari et al in Iran, expression of Teriparatide was approximately $43 \%$ of whole protein expression that was less than the present study (16).

Teriparatide is a small polypeptide that has been mostly studied in fusion with other proteins like NUS
A, IgG and MBP for prevention of digestion by host proteases. In our study, Trx fused expression of Teriparatide was investigated in two hosts, including Rosetta-gami and BL21 (17). In addition, this molecular tag increased the expression level of Teriparatide by causing soluble expression of this product instead of the inclusion form, as well as, preventing deformation of protein structure in the cytoplasm by effecting on disulfide bindings leading to reduction in downstream procedures and hence save a lot in costs. Our results showed that the Teriparatide expression was $45 \%$ of the total protein in Rosetta-gami that was more in comparison with the BL21 and other surveys.

Using the molecular labels was almost unavoidable in the expression process of many recombinant proteins, while, the presence of some excess factors in the final products was used of a drug is impossible. Thus, detachment of adjunct factors of the main product after purification is important in recombinant drug producing process. Enterokinase enzymes, by cutting the specific sites with no addition of any amino acids to the product and without harm or effect to humans, are one of the most common ways for detachment of the protein labels. Therefore, enterokinase was used to obtain $4 \mathrm{kDa}$ rhPTH purified product(18).

According to presented method in this study, the efficiency of the fused form of rhPTH expression is equal to $26 \mathrm{mg} / \mathrm{liter}$ of culture media of which $70 \%$ was purified. This yield is a suitable output compared to the other investigations and can be improved by using fermenter system and can be used as a drug candidate for Osteoporosis therapy following the functional evaluation of purified protein in both in vitro and in vivo. Since this product was obtained in soluble form and in proper concentration by using the mentioned procedure, it can be applied as an appropriate way for producing this polypeptide.

\section{Acknowledgments}

This article was supported by research center for Biosciences and Biotechnology, Malek Ashtar University of Tehran.

\section{Author contributions}

FB and HR designed and directed the project, SY and MK performed the experiments, SY and FB wrote the article.

\section{Conflict of Interest}

There is no Conflict of Interest.

\section{Support/Funding}

This work was supported by Malek-Ashtar University. 


\section{References}

1. Jin L BS, Chandrasekhar S. crystal structure of human parathyroid hormone 1-34 at 0.9-A resolution. J Biol Chem. 2000; 275:27238-44. PMID: 10837469

2. Schluter KD WM, Piper HM. parathyroid hormne induces protein kinase $\mathrm{C}$ but not adenylate cyclase in adult cardiomyocytes and regulates cyclic AMP levels via protein kinase $\mathrm{C}$ dependent. Biochem J. 1995; 310:439-44. PMID: 7654180

3. Sugiyama $\mathrm{T}$, Torio $\mathrm{T}$, Miyajima $\mathrm{T}$, Kim YT, Oda $\mathrm{H}$ Romosozumab and blosozumab: alternative drugs of mechanical strain-related stimulus toward a cure for osteoporosis. Frontiers Endocrinol. 2015; 6:54.

4. Ebina K, Hashimoto J, Kashii M, Hirao M, Kaneshiro S, Noguchi T, et al. The effects of switching daily teriparatide to oral bisphosphonates or denosumab in patients with primary osteoporosis. J Bone Miner Metab. 2017; 35(1):91-8. PMID: 26762133

5.Partridge NC, Li X, Qin L. Understanding Parathyroid Hormone Action. Ann N Y Acad Sci. 2006; 1068: 187-93. PMID: 16831918

6. Vad R NE, Dahl LA. Engineering of a Pichia pastoris expression system for secration of high amounts of intact human parathyroid hormone. J Biotechnol. 2005; 116(3):251-60. PMID: 15707686

7. Murby M CL, Nilsson J. Stabilization of recombinant proteins from proteolytic degradation in E. coli. Biotechnol Appl Biochem. 1991; 14(3): 336-46. PMID: 1777118

8. Suzuki Y, Yabuta M, Ohsuye K. High level production of recombinant human parathyroid hrmone 1-34. Appl Environ Microb. 1998; 64(2):526-9. PMID: 9464388

9. Fu XY, Tong WY, Wei DZ. Extracellular production of human parathuroid hormone as a thioredoxin fusion form in E. coli by chemical permeabilization combined with heat treat. Biotech Prog 2005; 21(5):1429-35. PMID: 16209546

10. He X, Li L, Xu H, Xi J, Cao X, Rong S, et al. A rice jacalin-related mannose-binding lectin gene, OsJRL, enhances Escherichia coli viability under high salinity stress and improves salinity tolerance of rice. Plant Biol. 2017; 19(2):257-67.
11. Arnau J, Lauritzen C, Petersen GE, Pedersen J. Current strategies for the use of affinity tags and tag removal for the purification of recombinant proteins. Protein Expr Purif. 2006; 48(1): 1-13. PMID: 16427311

12. Heras J, Dominguez C, Mata E, Pascual V, Lozano C, Torres, and Zarazaga M. GelJ - a tool for analyzing DNA fingerprint gel images. BMC Bioinformatics. 2015; 16: 270. PMID: 26307353

13. Grover HS, Luthra S, Maroo S. Teriparatide: A Novel Means to Ultimately Achieve True Regeneration. J Clin Diagn Res. 2013; 7(8): 1820-3. PMID: 24086927

14. Forsberg G, Brobjer M, Holmgren E, Bergdahl K, Persson P, Gautvik KM, et al. Thrombin and H64A subtilisin cleavage of fusion proteins for preparation of human recombinant parathyroid hormone. J Protein Chem. 1991; 10(5): 517-26. PMID: 1799410

15. Liu Q, Lin J, Liu M, Tao X, Wei D, Ma X, et al. Large scale preparation of recombinant human parathyroid hormone 1-84 from Escherichia coli. Protein Expr Purif. 2007; 54(2): 212-9. PMID: 17449274

16. Bakhtiari N Amini Bayat Z, Sagharidouz S, Vaez M. Overexpression of Recombinant Human Teriparatide, rhPTH (1$34)$ in Escherchia coli: an innovative gene fusion approach. Avicenna J Med Biotechnol. 2017; 9(1): 19-22. PMID: 28090276

17. Hamedifar H, Salamat F, Saffarion M, Ghiasi M, Hosseini A, Lahiji $\mathrm{H}$, et al. A novel approach for high level expression of soluble recombinant human parathyroid hormone (rhPTH 1-34) in E. coli. Avicenna J Med Biotechnol. 2013; 5(3): 193-201. PMID: 23919123

18. Edward RL, Elizabeth AD, Sharlotte K, Kathleen LG, Paul FS, John MM. A tioredoxin gene fusion expression system that circumvents inclusion body formation in the E.coli cytoplasm. Bitechnol. 1993; 11: 187-93. 